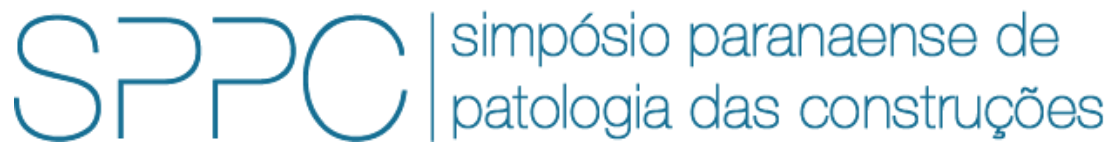

ISSN 2526-7248 artigo 2SPPC1006 pp. 57-66, 2017

\title{
Análise do processo de inspeção de pontes utilizando a mineração de processos
}

\author{
Eduarda Lauck Machado', Eduardo Alves Portela Santos ${ }^{2}$ e Adriana de Paula Lacerda Santos ${ }^{3}$ \\ ${ }^{1}$ Mestranda do Programa de Pós-Graduação em Engenharia de Construção Civil, Universidade \\ Federal do Paraná (UFPR), eduarda.lauck@ufpr.br \\ ${ }^{2}$ Programa de Pós-Graduação em Engenharia de Produção e Sistemas, Pontifícia Universidade \\ Católica do Paraná (PUCPR), eduardo.portela@pucpr.br \\ 3 Programa de Pós-Graduação em Engenharia de Produção, Universidade Federal do Paraná \\ (UFPR), adrianapls1@gmail.com
}

Resumo: A gestão de pontes lida principalmente com a priorização das necessidades de manutenção, planejamento dos procedimentos para realizar a manutenção como também a otimização do custo do ciclo de vida da ponte. Neste contexto, as técnicas de mineração de processos apresentam-se como uma contribuição ao procedimento de inspeção, uma vez que permitem extrair informações de processos por meio de logs de eventos, conhecer o processo e propor melhorias. Este artigo teve como objetivo analisar um processo de Inspeção de Pontes, utilizando a mineração de processos para identificar informações importantes e contribuir para o aprimoramento das atividades de vistoria de pontes de concreto armado. Observou-se que não há variações preocupantes no processo, apesar da necessidade de controlar o registro das atividades para obter uma análise mais precisa do processo.

Palavras-chave: Inspeção de pontes. Mineração de Processos. Projeto de Mineração.

Abstract: Bridge management mainly deals with the prioritization of maintenance needs, planning of the procedures to carry out the maintenance as well as the optimization of the cost of the life cycle of the bridge. In this context, process mining techniques are presented as a contribution to the inspection procedure, since they allow extracting information from processes through event logs, knowing the process and proposing improvements. This article aims to analyze the bridge's inspection activity log, by using the processes mining, in order to identify important information in this process and contribute to the improvement of inspection activities of reinforced concrete bridges. It was noted that there are no worrying variations in the process, despite the need to control the record of activities to get a more accurate analysis of the process.

Keywords: Inspection of bridges. Process Mining. Mining Project. 
MACHADO, E. L.; SANTOS, E. A. P.; SANTOS, A. P. L., ANÁLISE DO PROCESSO DE INSPEÇÃO DE PONTES UTILIZANDO A MINERAÇÃO DE PROCESSOS. $2^{\circ}$ Simpósio Paranaense de Patologia das Construções (2० SPPC), artigo 2SPPC1006, pp. 57-66,

\section{Introdução}

As pontes são componentes importantes em redes rodoviárias e demandam as atividades de manutenção corretiva e preventiva. Tais estruturas se deterioram com o tempo devido à fadiga, efeitos ambientais e possível sobrecarga [1-2]. Mais recentemente as pesquisas se focaram no tema da gestão eficiente de estruturas de pontes e no desenvolvimento de sistemas de gestão de ponte para auxiliar os decisores na criação de programas de reparação e manutenção eficientes [3]. Porém, para se alcançar um bom sistema de gerenciamento de pontes, é necessária a confiabilidade da técnica adotada para o diagnóstico de deterioração.

A gestão de pontes lida principalmente com a priorização das necessidades de manutenção, planejamento dos procedimentos para realizar a manutenção como também a otimização do custo do ciclo de vida da ponte. Entre as principais funções da gestão de uma ponte estão a avaliação do estado da ponte e a determinação de tratamentos disponíveis [1].

Quando um componente de uma ponte atinge um nível crítico em termos de desempenho e segurança, uma ação é necessária para restaurar ou atualizar a condição do componente. Entre as ações disponíveis estão a manutenção, reparo e substituição para os elementos da ponte. As agências de transporte têm de lidar com um número crescente de pontes deficientes que carecem de intervenção, que são ainda itens caros para estas agências [4].

O Departamento Nacional de Infraestrutura de Transportes (DNIT) é responsável pela gestão de 5.114 viadutos e túneis, as chamadas Obras de Artes Especiais (OAEs) [5]. Assim, é de grande importância otimizar os trabalhos de manutenção e reparação de pontes tanto para solicitar o financiamento adequado, quanto para usar de modo eficiente os recursos públicos [6].

Neste contexto, as técnicas de mineração de processos apresentam-se como uma contribuição ao procedimento de inspeção, uma vez que permitem extrair informações de processos por meio de logs de eventos, conhecer o processo e propor melhorias [7].

Desta forma, este artigo tem como objetivo, através de um estudo de caso, analisar um processo de Inspeção de Pontes, utilizando a mineração de processos para identificar informações importantes e contribuir para o aprimoramento das atividades de vistoria de pontes de concreto armado.

\section{A mineração de processos}

A mineração de processos (process mining, termo mais comum em inglês) compreende técnicas, ferramentas e métodos para descobrir, monitorar e melhorar os processos que de fato ocorreram, extraindo conhecimento a partir de logs de eventos comumente disponíveis nos sistemas de informação [7]. Ainda segundo os autores, a mineração de processos pode ser dividida em três tipos: descoberta, conformidade e melhoria. Na Fig. 1, tem-se o posicionamento dos três principais tipos de mineração de processo. 
MACHADO, E. L.; SANTOS, E. A. P.; SANTOS, A. P. L., ANÁLISE DO PROCESSO DE INSPEÇÃO DE PONTES UTILIZANDO A MINERAÇÃO DE PROCESSOS. $2^{\circ}$ Simpósio Paranaense de Patologia das Construções (2 SPPC), artigo 2SPPC1006, pp. 57-66,

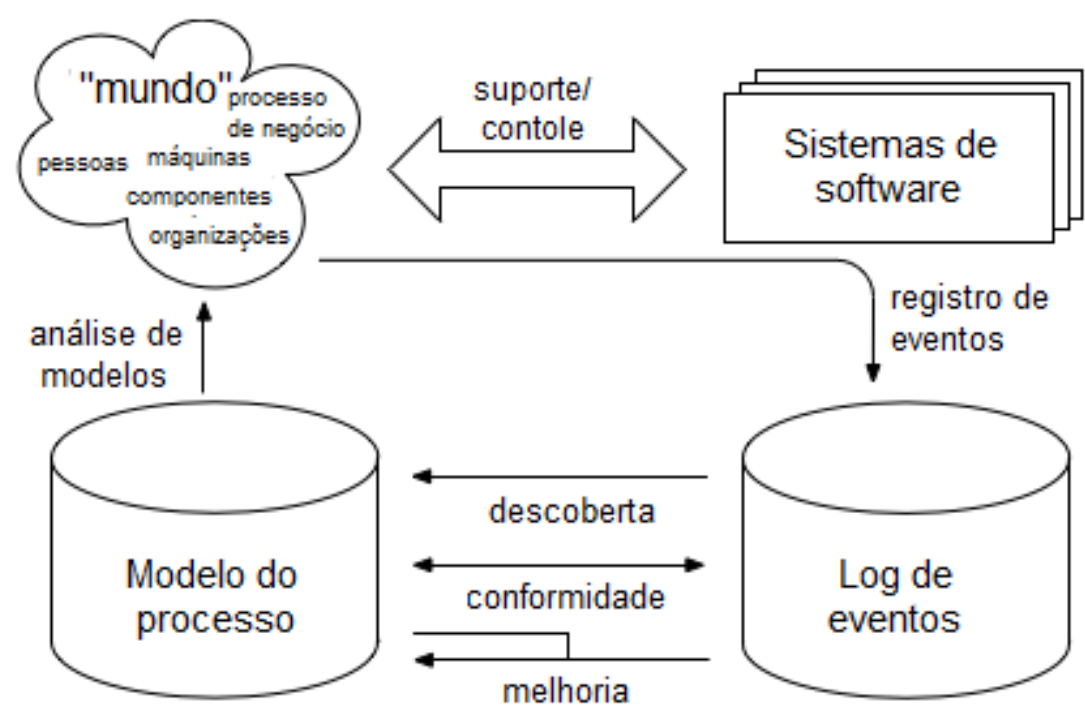

Figura 1: Posicionamento dos três principais tipos de mineração de processo. Adaptado de VAN DER AALST et al. [7].

Na descoberta, um log de eventos é convertido em um modelo de processo, sem o uso de informações adicionais. No segundo tipo, a conformidade, um modelo de processo existente é comparado com um log de eventos do mesmo processo, proporcionando assim a verificação de conformidade entre a realidade (o que é registrado no log) e o modelo e vice-versa. No terceiro tipo, a melhoria, a ideia é estender ou melhorar um modelo de processo já existente usando informações sobre o processo real (aquele que foi registrado).

Uma das formas de se executar a mineração de um processo é por meio de um projeto de mineração. Para isso, existem vários métodos e entre eles o $\mathrm{PM}^{2}$, que é uma metodologia de projeto de mineração de processos (Process Mining Project Methodology) que abrange uma vasta gama de outras técnicas de análise de processo e de mineração, e é adequado para a análise de processos estruturados e não estruturados [8]. Na Fig. 2 apresenta-se uma visão geral deste método, que será utilizado para a mineração do processo de inspeção de pontes.

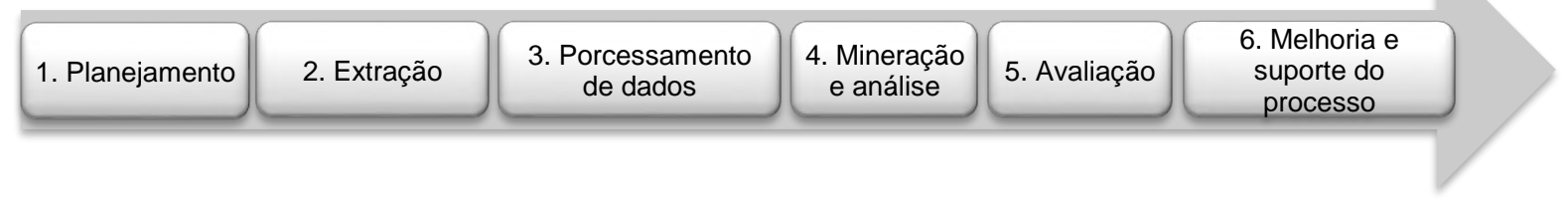

Figura 2: Visão geral do método $\mathrm{PM}^{2}$. Adaptado de VAN ECK et al. [8].

O objetivo da fase de planejamento é configurar o projeto e determinar as questões de pesquisa. Esta fase possui três atividades: identificar questões de pesquisa, selecionar os processos de negócios e compor a equipe de projeto.

A fase de extração tem por objetivo extrair dados de eventos como também os modelos de processo, e as atividades para essa fase são: determinar o escopo, extrair os dados de eventos, e transferir o conhecimento do processo. 
MACHADO, E. L.; SANTOS, E. A. P.; SANTOS, A. P. L., ANÁLISE DO PROCESSO DE INSPEÇÃO DE PONTES UTILIZANDO A MINERAÇÃO DE PROCESSOS. $2^{\circ}$ Simpósio Paranaense de Patologia das Construções (2० SPPC), artigo 2SPPC1006, pp. 57-66, 2017. DOI: $10.4322 / 2$ SPPC. 2017.006

Já a fase de processamento de dados tem como objetivo criar logs de eventos como diferentes vistas, e possui quatro tipos de atividades: criação de vistas, integração de eventos, enriquecimento e filtragem dos logs.

$\mathrm{Na}$ fase de mineração e análise, utiliza-se as técnicas de mineração de processo nos logs de eventos e esta fase tem como objetivo responder às questões de pesquisa e ter uma visão sobre o desempenho do processo e conformidade. As quatro atividades que compõem essa fase são: descoberta do processo, verificação da conformidade, aprimoramento e análise do processo.

A fase de avaliação tem como objetivo relacionar os resultados da análise com as ideias de melhoria que permitam atingir os propósitos do projeto, e as atividades desta fase são: diagnóstico, e verificação e validação.

\section{Inspeção de pontes}

O processo a ser analisado neste estudo de caso compreende as etapas de PréInspeção, Inspeção propriamente dita e Pós-Inspeção, que têm o objetivo de padronizar o planejamento e o procedimento para a realização das verificações das estruturas, de modo a minimizar os erros referentes à subjetividade e às opiniões particulares dos avaliadores, alcançando assim uma maior confiabilidade dos relatórios de vistorias. Cada uma dessas etapas tem procedimentos prédeterminados que podem ser visualizados na Fig. 3.

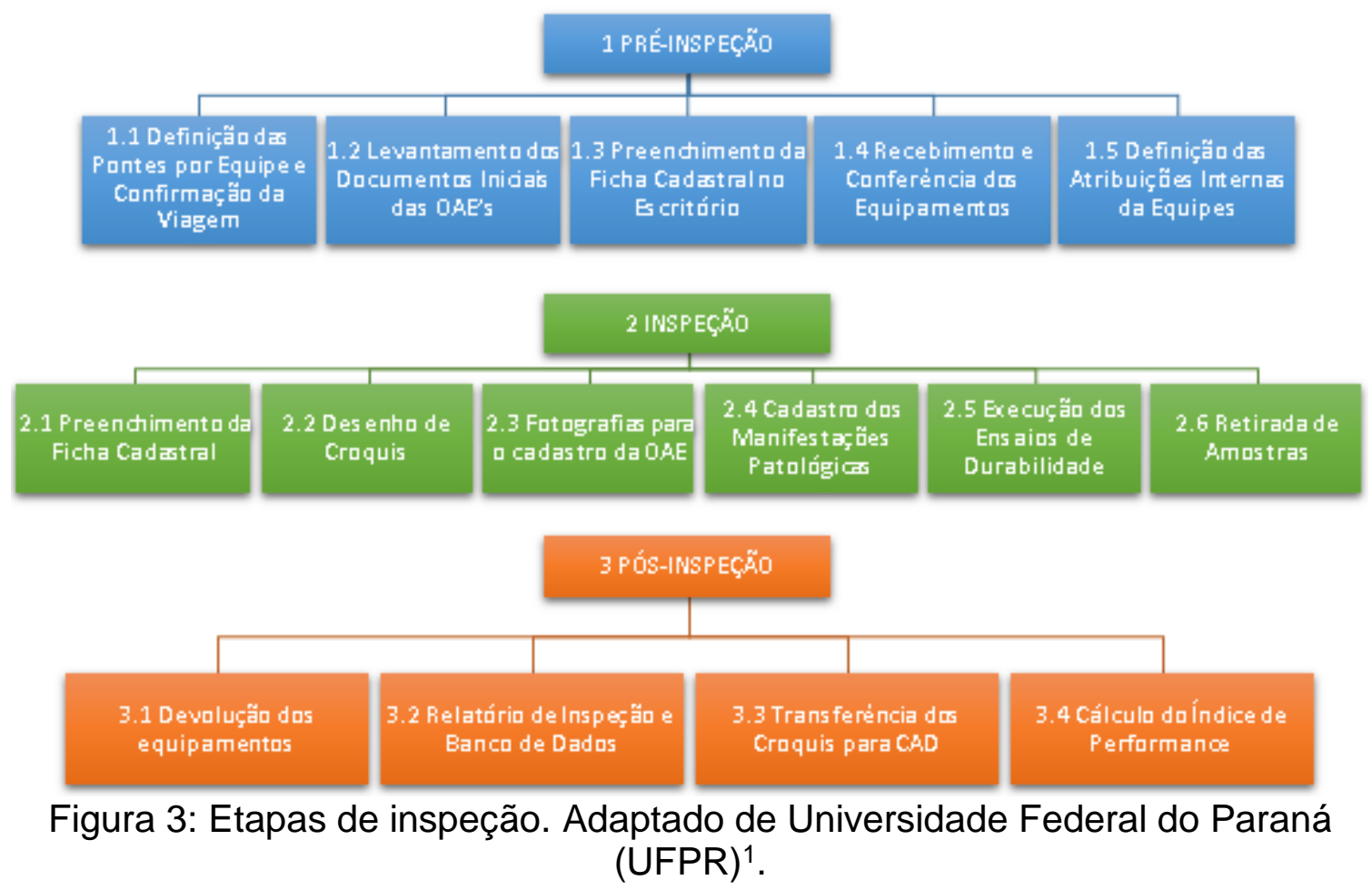

Na primeira etapa da pré-inspeção, que se refere à definição das pontes por equipes e confirmação da viagem, as equipes de vistoria se organizam, duas semanas antes,

\footnotetext{
${ }^{1}$ Universidade Federal do Paraná (UFPR). METODOLOGIA: Diretrizes para inspeção de Obras de Arte Especiais. Vol. III. Em fase de elaboração.
} 
MACHADO, E. L.; SANTOS, E. A. P.; SANTOS, A. P. L., ANÁLISE DO PROCESSO DE INSPEÇÃO DE PONTES UTILIZANDO A MINERAÇÃO DE PROCESSOS. $2^{\circ}$ Simpósio Paranaense de Patologia das Construções (2० SPPC), artigo 2SPPC1006, pp. 57-66,

para confirmar integrantes da viagem e a quantidade de pontes a serem inspecionadas, como também verificação de dados fundamentais antes da saída para inspeção como: localização das pontes (coordenadas, $\mathrm{km}$ ), hotéis e restaurantes mais próximos e meio de transporte adequado aos inspetores e para os equipamentos de inspeção. Na etapa de levantamento dos documentos iniciais das pontes, as equipes se ocupam do conhecimento/acesso a documentos (projetos, inspeções anteriores) de órgãos responsáveis pelas pontes. Antes de realizar a inspeção propriamente dita, os projetos encontrados na etapa anterior são estudados e as informações são documentadas numa planilha de pré-cadastro, de forma a registrar as informações contidas nos projetos, verificando posteriormente se elas condizem com o executado. $\mathrm{Na}$ sexta-feira prévia a inspeção, as equipes preenchem os dados do checklist dos equipamentos necessários à inspeção, para retirada dos equipamentos. Ainda na semana prévia à inspeção é feita a divisão das atividades de acordo com as atribuições de cada integrante.

Já na inspeção, o primeiro procedimento é o preenchimento da ficha cadastral da ponte. Para o desenho dos croquis, deve ser seguida a sequência de desenho e a identificação dos elementos com a nomenclatura já estabelecidas. As fotografias para o cadastro da ponte, que devem ser tiradas durante a inspeção, necessitam seguir também as orientações já estabelecidas. Na sequência é feito o cadastro das manifestações patológicas encontradas nas pontes, para a posterior análise e ranqueamento das mesmas. Durante a inspeção devem ser feitos também alguns ensaios como de esclerometria, de potencial de corrosão e de carbonatação.

Na semana após a inspeção, começa a primeira etapa de pós-inspeção, que consiste na devolução dos equipamentos utilizados nas vistorias. Em seguida, todos os dados são adequadamente organizados no relatório de inspeção e no banco de dados da ponte inspecionada. Também é feita a transferência dos croquis desenhados em campo para um croqui digital em formato ".dwg". Finalmente é realizado o Cálculo do Índice de Performance, que servirá de parâmetro para avaliação do estado das estruturas.

Neste artigo, será analisado o registro dessas atividades, realizadas no mês de julho de 2016, com o objetivo de identificar informações importantes neste processo, e contribuir para a melhoria das atividades de vistoria de pontes de concreto armado.

\section{Método}

Para realizar a mineração do processo de inspeção de pontes será realizado um estudo de caso, seguindo o método $\mathrm{PM}^{2}$, utilizando o software de mineração Disco, que possui uma estrutura de gerenciamento e filtragem de log eficiente, e cuja plataforma é fácil de usar e permite uma boa visualização dos mapas do processo.

\subsection{Planejamento}

$\mathrm{Na}$ fase de planejamento foi definido como objetivo conhecer o processo de inspeção de pontes de concreto armado, de modo a identificar informações importantes contribuindo para a melhoria do processo. Foi também proposto a utilização de uma planilha eletrônica como meio para registro dos eventos de inspeção. Na Fig. 4, temse um trecho da planilha utilizada pela equipe de inspeção. 
MACHADO, E. L.; SANTOS, E. A. P.; SANTOS, A. P. L., ANÁLISE DO PROCESSO DE INSPEÇÃO DE PONTES UTILIZANDO A MINERAÇÃO DE PROCESSOS. $2^{\circ}$ Simpósio Paranaense de Patologia das Construções (2० SPPC), artigo 2SPPC1006, pp. 57-66, 2017. DOI: $10.4322 / 2$ SPPC. 2017.006

\begin{tabular}{|c|c|c|c|c|c|c|}
\hline \multirow{2}{*}{$\begin{array}{c}\text { Identificação } \\
\text { da ponte }\end{array}$} & \multicolumn{2}{|c|}{ Início } & \multicolumn{2}{c|}{ Término } & & \\
\cline { 2 - 7 } & Data & Hora & Data & Hora & Etapa & Responsável \\
\hline $427 . a$ & $18 / 07 / 2016$ & $15: 00$ & $18 / 07 / 2016$ & $15: 30$ & 2.1 & INTEGRANTE 01 \\
\hline $427 . a$ & $18 / 07 / 2016$ & $15: 00$ & $18 / 07 / 2016$ & $15: 45$ & 2.2 & INTEGRANTE 02 \\
\hline $427 . a$ & $18 / 07 / 2016$ & $15: 00$ & $18 / 07 / 2016$ & $15: 50$ & 2.3 & INTEGRANTE 03 \\
\hline $427 . a$ & $18 / 07 / 2016$ & $15: 30$ & $18 / 07 / 2016$ & $16: 00$ & 2.4 & INTEGRANTE 04 \\
\hline
\end{tabular}

Figura 4: Trecho da planilha utilizada para registro dos eventos. Os autores.

\subsection{Extração}

Para a extração dos dados dos eventos e visualização do processo foi utilizado o software Disco e foram consideradas como importantes as informações relacionadas ao tempo de execução e das atividades.

\subsection{Processamento de dados}

Assim como a extração dos dados dos eventos, o processamento de dados foi realizado utilizando o Disco. Por meio deste software, obteve-se o mapa do processo de inspeção, e foram aplicados filtros relacionados aos eventos, agrupando-os por etapa de inspeção, e filtros relacionados ao tempo médio que as etapas de inspeção demoraram para serem completadas.

\subsection{Mineração e análise}

A atividade de descoberta do processo, apresentada na seção seguinte, foi realizada através da aplicação dos filtros mencionados anteriormente. Já a atividade de verificação da conformidade não pode ser executada devido à limitação dos dados, deste modo, as sugestões de melhoria deste processo serão baseadas nestas limitações.

\subsection{Avaliação}

$\mathrm{Na}$ fase de avaliação procurou-se então, associar os resultados da análise de mineração com propostas de melhorias para o processo de inspeção.

\subsection{Melhoria e Suporte do Processo}

Nesta fase procura-se apresentar sugestões de melhorias para um novo projeto de mineração de modo que se possa obter melhores resultados na análise do processo, como também no processo de inspeção.

\section{Resultados}

Acessando os registros das vistorias utilizando o software Disco e analisando as informações iniciais do processo tem-se as seguintes informações:

- Número de atividades executadas pelo registro: 242

- Número de casos registrados: 22

- Número de atividades ou eventos: 11

o Recursos envolvidos nessas atividades: 5 
MACHADO, E. L.; SANTOS, E. A. P.; SANTOS, A. P. L., ANÁLISE DO PROCESSO DE INSPEÇÃO DE PONTES UTILIZANDO A MINERAÇÃO DE PROCESSOS. $2^{\circ}$ Simpósio Paranaense de Patologia das Construções (2० SPPC), artigo 2SPPC1006, pp. 57-66, 2017. DOI: $10.4322 / 2$ SPPC.2017.006

Observa-se ainda que as inspeções iniciaram com a atividade de "Definição das Pontes por Equipe e Confirmação da Viagem" e terminaram com "Transferência dos Croquis para CAD”, e tiveram uma média de 11 dias de duração.

$\mathrm{Na}$ Fig. 5 apresenta-se o mapa de atividades com suas respectivas frequências absolutas de ocorrência no processo. Neste processo observa-se apenas um padrão de sequência de atividades que ocorre sem repetições. Apresenta-se ainda, a duração atividades, bem como o tempo médio entre elas.

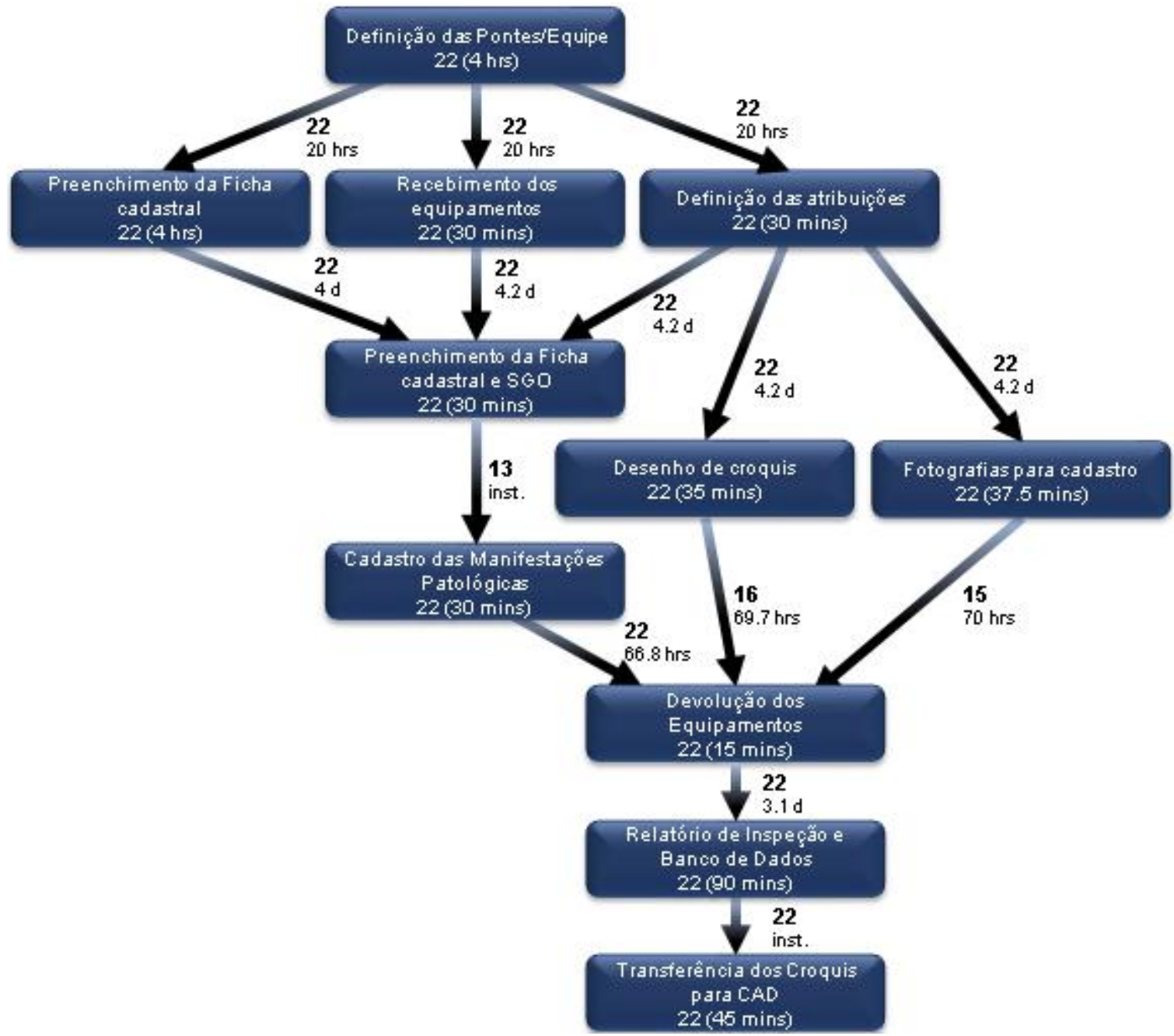

Figura 5: Mapa do processo de Inspeção com a duração média das atividades e tempo entre elas. Os autores.

O fato deste processo apresentar apenas um padrão de atividades e estas seguirem a sequência pré-estabelecida, indica que as vistorias ocorrem conforme o planejado não ocorrendo variações indesejadas.

A partir da Fig. 6 observa-se também que o tempo de espera entre as atividades varia entre de 2 dias e 14 horas a 3 dias e 2 horas. O que pode ser atribuído aos recessos de finais de semana e tempo de viagem das equipes de inspeção, não configurando gargalos no processo. 
MACHADO, E. L.; SANTOS, E. A. P.; SANTOS, A. P. L., ANÁLISE DO PROCESSO DE INSPEÇÃO DE PONTES UTILIZANDO A MINERAÇÃO DE PROCESSOS. $2^{\circ}$ Simpósio Paranaense de Patologia das Construções (2० SPPC), artigo 2SPPC1006, pp. 57-66, 2017. DOI: $10.4322 / 2$ SPPC.2017.006

Filter cases by: Mean waiting time $\rightarrow$

Extend range

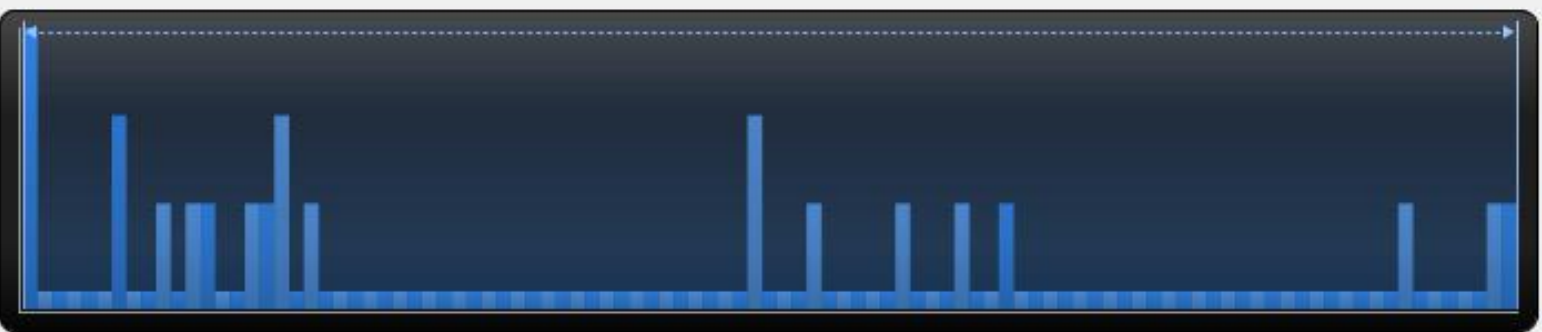

\section{2 days, 14 hours $\quad *$ \\ Minimum waiting time}

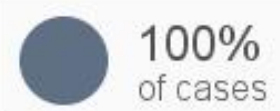

of cases

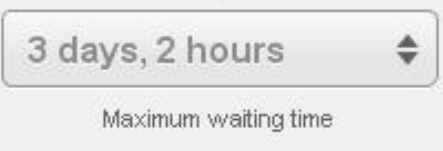

Figura 6: Tempo de espera entre as atividades de Inspeção. Os autores.

Utilizando um filtro de atributos e selecionando apenas as atividades da pré-inspeção, observa-se que para todas as pontes que foram registradas, a duração total de execução desta etapa é de 28 horas, sendo que as atividades de "Definição das Pontes por Equipe e Confirmação da Viagem" e "Preenchimento da Ficha Cadastral no Escritório" têm duração total de 4 horas cada, enquanto as atividades de "Recebimento e Conferência dos Equipamentos" e "Definição das Atribuições Internas da Equipe", são atividades mais rápidas, com duração de 30 minutos.

Numa filtragem de atributos, selecionando apenas as atividades da Inspeção em campo, (Preenchimento da Ficha Cadastral e SGO", "Desenho de Croquis", "Fotografias para o cadastro da OAE" e "Cadastro das Manifestações Patológicas") tem-se que cada uma delas é desenvolvida por apenas um integrante e têm em média de 30 a 40 minutos de duração (Fig. 7).

\begin{tabular}{|l|r|r|r|}
\hline Resource & A Frequency & Relative frequency & Median duration \\
\hline Integrante01 & 22 & $25 \%$ & 30 mins \\
Integrante02 & 22 & $25 \%$ & 35 mins \\
Integrante03 & 22 & $25 \%$ & 37 mins, 30 secs \\
Integrante04 & 22 & $25 \%$ & 30 mins \\
\hline
\end{tabular}

Figura 7: Duração média das atividades da Inspeção em campo, por integrante. Os autores.

Analisando então apenas as atividades de Pós-Inspeção, por meio de um filtro de atributos, observa-se que esta etapa leva em média de 3 dias para ser concluída e 0 tempo de duração média de cada uma delas pode ser visualizado na Fig. 8.

\begin{tabular}{|l|r|r|r||}
\hline Activity & Frequency & Relative frequency & Median duration \\
\hline Devolução dos Equipamentos & 22 & $33,33 \%$ & 15 mins \\
Relatório de Inspeção e Banco de Dados & 22 & $33,33 \%$ & 1 hour, 30 mins \\
Transferência dos Croquis para CAD & 22 & $33,33 \%$ & \\
\hline
\end{tabular}

Figura 8: Duração média das atividades de Pós-Inspeção. Os autores. 
MACHADO, E. L.; SANTOS, E. A. P.; SANTOS, A. P. L., ANÁLISE DO PROCESSO DE INSPEÇÃO DE PONTES UTILIZANDO A MINERAÇÃO DE PROCESSOS. $2^{\circ}$ Simpósio Paranaense de Patologia das Construções (2० SPPC), artigo 2SPPC1006, pp. 57-66,

A partir destas análises, é possível dizer que os integrantes das equipes de inspeção não estão sobrecarregados, uma vez que executam tarefas distintas em etapas distintas.

As atividades de "Levantamento dos Documentos Iniciais das OAE's", "Execução dos Ensaios de Durabilidade", "Retirada de Amostras" e "Cálculo do Índice de Performance" não aparecem no modelo do processo por não terem sido registradas no log. Tal fato, a princípio não se caracteriza como um erro no processo, uma vez que podem ter sido executadas em um momento anterior ao início dos registros, como é o caso, por exemplo da atividade de "Levantamento dos Documentos Iniciais das OAE's". Já as atividades de "Execução dos Ensaios de Durabilidade" e "Retirada de Amostras" podem não ter sido executadas por não se aplicar a inspeção em questão, uma vez que as pontes poderiam se encontrar em bom estado de conservação. A atividade de "Cálculo do Índice de Performance" possivelmente não se encontra no log por estar em andamento.

Para obter resultados melhores na análise de procedimentos de vistoria de pontes, sugere-se um novo projeto de mineração, envolvendo um tempo maior de investigação, com foco na conscientização da equipe quanto ao registro completo das atividades, para que a mineração possa ser realizada no processo inteiro.

\section{Conclusões}

O principal objetivo deste trabalho foi, através de um estudo de caso, analisar um processo de Inspeção de Pontes utilizando um projeto de mineração de processos, de modo a identificar informações importantes do procedimento, e contribuir para melhorias das atividades de vistoria das pontes.

Após a análise do processo utilizando o software Disco, conclui-se que as atividades são executadas como planejado e no tempo esperado, sendo que não há variações preocupantes no processo analisado.

Sugere-se ainda, para melhores resultados na análise de procedimentos de vistoria de pontes, que seja feito um registro mais rigoroso das atividades, de modo que possa ser realizada uma análise completa do processo, incluindo a etapa de análise de conformidade com suas propostas de melhorias.

\section{Agradecimentos}

Agradecemos ao Escritório Modelo de Engenharia Civil (EMEA) da Universidade Federal do Paraná, que vem desenvolvendo os trabalhos de inspeções das pontes, nos estados do Paraná e de Santa Catarina, e disponibilizou os dados para o desenvolvimento deste artigo.

\section{Referências}

[1] Chassiakos, A. P.; Vagiotas, P.; Theodorakopoulos, D. D. (2005) A knowledgebased system for maintenance planning of highway concrete bridges, Advances in Engineering Software, 36 (11-12), 740-749. 
MACHADO, E. L.; SANTOS, E. A. P.; SANTOS, A. P. L., ANÁLISE DO PROCESSO DE INSPEÇÃO DE PONTES UTILIZANDO A MINERAÇÃO DE PROCESSOS. $2^{\circ}$ Simpósio Paranaense de Patologia das Construções (20 SPPC), artigo 2SPPC1006, pp. 57-66, 2017. DOI: $10.4322 / 2$ SPPC. 2017.006

[2] Sasmal, S.; Ramanjaneyulu, K. (2008) Condition evaluation of existing reinforced concrete bridges using fuzzy based analytic hierarchy approach. Expert Systems with Applications, 35 (3),1430-1443.

[3] Zhao, Z.; Chen, C. (2002) A fuzzy system for concrete bridge damage diagnosis. Computers and Structures, 80 (7-8), 629-641.

[4] Abu Dabous, S; Alkass, S (2008). Decision support method for multi-criteria selection of bridge rehabilitation strategy, Construction Management and Economics, 26 (8), 883-893.

[5] Lee, J. et al. (2008) Improving the reliability of a Bridge Management System (BMS) using an ANN-based Backward Prediction Model (BPM). Automation in Construction, 17 (6), 758-772.

[6] Departamento Nacional de Infraestrutura de Transportes (DNIT). Pontes e viadutos das rodovias federais estão em boas condições. Disponível em: http://www.dnit.gov.br/noticias/pontes-e-viadutos-das-rodovias-federais-estaoem-boas-condicoes. Acessado em: 04 jan. 2017.

[7] Van der aalst, W. et al. Process Mining Manifesto. In: Daniel, F.; Barkaoui, K.; Dustdar, S. (Eds.). Business Process Management Workshops: BPM 2011 International Workshops, Clermont-Ferrand, France, August 29, 2011, Revised Selected Papers, Part I. Berlin, Heidelberg: Springer Berlin Heidelberg, 2012, 169-194.

[8] Van eck, M. L. et al. PM2: A Process Mining Project Methodology. In: International Conference on Advanced Information Systems Engineering. Springer International Publishing, 2015, 297-313. 